\title{
Polymer Based Microchip for Combined Capillary Electrophoresis and Electrochemical Detection
}

\author{
M. Rashid, V. Auger, L. Williams, Y. Dou, S. Pruneanu and Z. Ali
}

\begin{abstract}
We have developed Microsystems with capillary electrophoresis and an electrochemical detector (CE-ECD). The microfabricated CE-ECD systems can be used as a disposable device and the characteristics have been optimized for application in electrochemical detection. The system has been realized on a Polystyrene (PS) polymer chip with gold electrodes. The injection and separation channels were produced by relatively simple and inexpensive methods. An electro-osmotic flow system (EOF) and a three-electrode electrochemical detector were fabricated on the same substrate in a single process. Cyclic voltammetry has been used to test the electrodes behaviour with catechol and dopamine in buffers of different $\mathrm{pH}$. In this article, we give an overview on the methodological aspects of coupling ED (electrochemical Detection) with Capillary Electrophoretic systems.
\end{abstract}

\section{INTRODUCTION}

Interest in micro analytical devices has grown immensely recently and opened new possibilities for the miniaturization of conventional chemical and biochemical analysis.[1-2]. The main potential of these microchips is to provide clinical diagnostic tests without the need of sending a sample to a laboratory for sample processing. [3] Capillary Electrophoresis (CE) in the microchip format was initially described in 1990 and has become more common place in research labs.[4-9]. The advantages of miniaturized systems are numerous and include speed of analysis, increased precision and accuracy, portability, increased throughput, reduced cost and waste, disposability, and the potential for on-site use. $[5,6,10]$. Due to the rapid expansion of the micro devices in analytical market, many researchers have

Manuscript received April 2, 2007

M. Rashid is with the Centre for Nano \& Microsystems, School of Science and Technology, University of Teesside, TS1 3BA, UK (e-mail: m.rashid@tees.ac.uk)

V. Auger is with the Centre for Nano \& Microsystems, School of Science and Technology, University of Teesside, TS1 3BA, UK (corresponding author - tel.: +44 1642342507; fax: +44 1642342401; email: v.auger@tees.ac.uk).

L. Williams is with the Institute of Pharmacy, Chemistry and Biomedical Sciences,University of Sunderland, Sunderland, SR1 3SD (email: Lee.williams@sunderland.ac.uk)

Y. Dou was in the School of Science and Technology, University of Teesside, TS1 3BA, UK She is now with the Electronics and Electrical Engg, University of Glasgow, Glasgow. G12 8LT. U.K (e-mail: yhdou@elec.gla.ac.uk)

S. Pruneanu was in the School of Science and Technology, University of Teesside, TS1 3BA, UK She is now with the School of Natural Sciences, University of Newcastle, Newcastle. NE1 7RU. U.K (e-mail: stela.pruneanu@newcastle.ac.uk)

Z. Ali is with the Centre for Nano \& Microsystems, School of Science and Technology, University of Teesside, TS1 3BA, UK (e-mail: z.ali@tees.ac.uk) developed a number of fabrication methods using low cost materials.[11-13].

Polymer based substrates have a number of advantages over quartz, silica and glass-based microchips. The polymer raw material is cheaper than silicon or glass and its processing involves mechanical or thermal processing, reducing the use of expensive chemical and limiting safety issues (wet and dry etching). However hydrophobic nature of the polymers reduces the ability to sustain electroosmotic flow (EOF) within channels and therefore constitutes a major obstacle to their deployment in certain device types; e.g. separation chips. Surface modification of the polymer surface and reducing the contact angle are the primary requirements of the manufacturing of microfluidic devices with the polymer substrate. [14-15]. Several research groups have used a number of polymer materials and have shown that polystyrene can be a good choice of substrate, having a good combination of chemical resistance to both the analytes and the running buffer $[13,16]$.

Types of detection in diagnostic applications depend strongly upon molecules of interest. Standard enzymatic and immunologic assays have been addressed recently for microchip applications $[14,15,17]$. Further advanced studies indicated the use of impedimetric measurements for specific proteins in complex biological sample [18, 19]. Several types of detection modes have been also employed to monitor separations on $\mathrm{CE}$ chip. Laser-induced fluorescence (LIF) has been the most popular mode for microchip CE due to its inherent high sensitivity.[20, 21]. However, most separated compounds are not naturally fluorescent and must be modified with a fluorophore in order to be detected by LIF. Conversely electrochemical detection can give high-sensitive detection with tunable selectivity (via applied voltage) and pre-defined conditions of detection. It also has the advantage in that it is a robust method of detection and thus is the ideal choice for cheap, portable and disposable devices. Among a wide range of electrochemical detection $[17,22]$, amperometric detection is attractive because of its high sensitivity, easily miniaturisable and compatible for Lab-on-Chip systems.

Among several biological targets, we focused the development of this CE-ECD microchip on two different neurotransmitters - Catechol and Dopamine - which present particular interest in both psychotic and metabolic human disorders [23-25]. Particularly both compounds are strongly related to Parkinson's syndrome [26]. Therefore the purpose of this study is to design, fabricate and characterize a simple 
polymer-based microchip for a stand-alone capillary electrophoresis microchip with an electrochemical detection.

\section{EXPERIMENTAL DETAILS}

\section{A. Chip design and instrumentation}

The CE microchip consists of two combined layers. The top layer contains the micro channel where the buffers and sample are injected by virtue of an EOF and where the sample components are separated according to their electrophoretic mobility. The bottom layer contains the microfabricated electrode layer where a set of 4 electrodes (EOF injection and EOF-ground in Figure 1) generates the various potential differences in order to produce the EOF and thus mobilize the fluids for injection and separation. A second set of 3 electrodes (WE, RE and CE in Figure 1) forms the electrochemical detection system. The high voltage power supply was obtained from CE resources Pte Ltd with integrated CE station software V5.8. The analysis system also contained a home made interlock box and an Auto lab analyzer.

\section{B. Fabrication of the separation channel}

$1.5 \mathrm{~mm}$ thick analytical grade polymer sheets were obtained from Intersign plc. The polymer sheets were cleaned by propan-2-ol followed by DI water. The top layer of CE chip was manufactured using a Hot Embosser (HEX 02, Jenoptik) $[11,27]$. The Al metal master embossing tool was patterned using a milling machine (Datron, PowerMill, 5.5) and was used to structure the micro channels in PS. During hot embossing, the $\mathrm{Al}$ metal alloy was heated $50^{\circ} \mathrm{C}$ above the $\mathrm{Tg}$ of the polystyrene polymer $\left(100^{\circ} \mathrm{C}\right)$ and high pressure $(15 \mathrm{kN})$ was applied to the PS substrate to transfer the desired micro channel structure in the PS. (Figure 1)

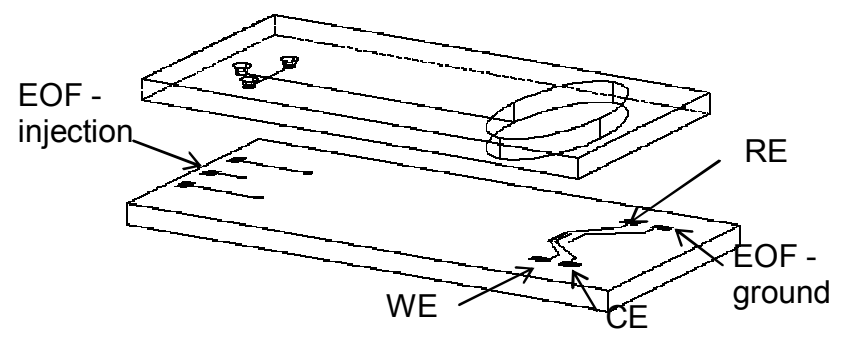

Figure 1: Exploded view of the top separation channel and the bottom EOF and EC electrodes.

The milling machine was used to produce the sample, buffer, waste sample and detection reservoirs. The diameter of sample, buffer and waste sample reservoirs was $1.5 \mathrm{~mm}$. The detection reservoir was milled just $20 \mu \mathrm{m}$ from the end of the separation channel. A connecting funnel (30 degrees opening) was milled between the separation micro channel and the detection reservoir to enhancing the decoupling performance.

Moulding and demoulding were performed with gradual temperature gradient, force and wait time. At the demoulding step, the wait time was $600 \mathrm{sec}$ with the gradual temperature decreases from $160^{\circ} \mathrm{C}$ to $70^{\circ} \mathrm{C}$. The electrophoresis channel was $50 \mu \mathrm{m}$ deep and $50 \mu \mathrm{m}$ wide. An interesting advantage of the hot-embossing is to provide rounded shape capillaries with square shape die due to the fluid polymer during the demoulding process.

\section{The electrode plate photolithography}

Electrode fabrication methods for microchips involve photolithography together with material deposition techniques, screen-printing techniques, laser ablation, wet chemistry and conventional manual microelectrode fabrication processes. We adapted the well established techniques of photolithography to polymers [28]. As a liftoff process needs stripping the photoresist away with acetone, we developed the process to replace the acetone by using a mixture of a stronger developer. An initial mixture of AZ400k (5:1 in water) was used for initial development. Thermal evaporator (Edwards Auto306 Resistance Evaporation System) was used for the deposition of the gold electrode on the polymer slide and a 3:1 mixture of developer (3:1) was used to lift off the remaining photoresist.

\section{Chip assembly}

An alignment mark was used to reproducibly align the PS channel layer with the electrode plate. The mark was aligned with the top of the electrode and ensured that a constant distance of $20 \mu \mathrm{m}$ between the end of the separation channel and the beginning of the detection reservoir.The separation channel layer (PS) was sealed to the PS electrode plate by bringing the two substrates into conformal contact with one another after extensive cleaning of the surfaces and drying with nitrogen. Thermal bonding techniques were applied for the final chip assembly [29]. A bonding temperature slightly above the $\mathrm{Tg}$ of polymer was applied with $5 \mathrm{~K}$ pressure and wait time 600 sec.

\section{E. Electrode modification}

An ideal electrochemical cell consists of different electrode materials for the working and reference electrodes and a pseudo reference $[17,22]$. In order to optimize the electrochemical signal in the system, we modified in-situ the gold reference electrodes into an $\mathrm{Ag} / \mathrm{AgCl}$ electrode.[30] The modification was carried out after the chip was assembled. A $2.0 \mathrm{mM} \mathrm{AgNO}_{3}$ solution was placed in the detection reservoir and a Pt external counter electrode was placed in the solution. The modification was cycled to a reduction potential $(0$ to- $0.4 \mathrm{~V}$ vs $\mathrm{Ag} / \mathrm{AgCl})$ for several minutes. The modification process was continued until the current had largely dissipated. Later, the $\mathrm{AgNO}_{3}$ solution was replaced with $1.0 \mathrm{mM} \mathrm{NaCl}$, and the potential was cycled back to $+0.4 \mathrm{~V}$. The process was continued until the oxidation current decreased to background.

\section{F. Sampling method}

Injection is an important issue with microchip CE-ED devices because the detection reservoir, which contains the working, auxiliary, and reference electrodes, connected to a potentiostat, must always be held earthed. Band broadening 
of the plugs can occur from leakage at the injection channel intersection.[31, 32] In our experimental detection system, we have introduced a gated injection system [17, 33]. Gated injections require voltage control of each reservoir. This means that one needs a separate voltage source for the sample, sample waste, and buffer reservoirs.

Figure 2 recaps the sampling and the separation sequence.

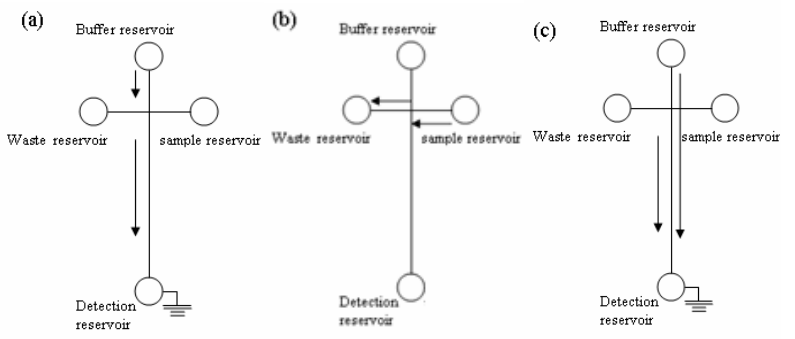

Figure 2: sampling sequence - (a)buffer reservoir connected, detection reservoir grounded, sample and waste reservoir floated.(b) Buffer and detection reservoir floated, sample reservoir connected and waste reservoir is grounded. (c) Sample and waste reservoir floated, buffer reservoir connected, detection reservoir grounded.

\section{G. Electrochemical characterization}

Separation and detection of the analytes on the CE chip depends significantly on the pKa values of the analytes and $\mathrm{pH}$ of the separation and sample buffers. We investigated the signal performance of the electrochemical detection using buffers at a range of $\mathrm{pH}$. We carried out standard cyclic voltametry with $5 \mathrm{mM}$ dopamine and catechol. A gold macro-electrode from BASi (Indiana, USA) was used as standard working electrode, with $\mathrm{Pt}$ as a counter electrode and $\mathrm{Ag} / \mathrm{AgCl}$ as the reference electrode. All the electrochemistry was performed using Auto lab software. Phosphate / acetate buffers at $\mathrm{pH}$ 3.93, 6.6 and 8.2 were used for the standard oxidation and reduction reaction. Initial trials were then carried out to assess the behavior of the onchip electrodes systems on the analysis of dopamine.

\section{RESUlts}

Catechol and dopamine are common analytes for CE micro chip separation and detection systems. Very low concentrations were used in the testing of the detection system, which used a standard oxidation/reduction reaction. Catechol and dopamine both act as neurotransmitters and they show almost identical electrochemical signals. So separation and detection of the catechol and dopamine using the CE-ED chip gives a challenging investigation.

Oxidation of dopamine and catechol was observed using a standard $\mathrm{Au}$ electrode, $\mathrm{Pt}$ counter electrode and $\mathrm{Ag} / \mathrm{AgCl}$ reference electrode. Cyclic Voltammetry was performed and the voltage was applied in the range: $-0.3 \mathrm{~V}$ to 0.75 , at
$100 \mathrm{mV} / \mathrm{sec}$. Buffers at several different $\mathrm{pH}$ values were used to see the variability of the electrochemistry reaction.
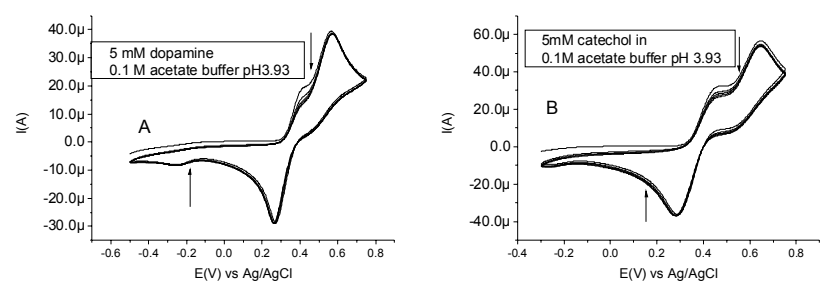

Figure 3: CV recorded in 5mM Dopamine (A) and catechol (B) in Acetate Buffer pH3.92 (Au macroelectrode)
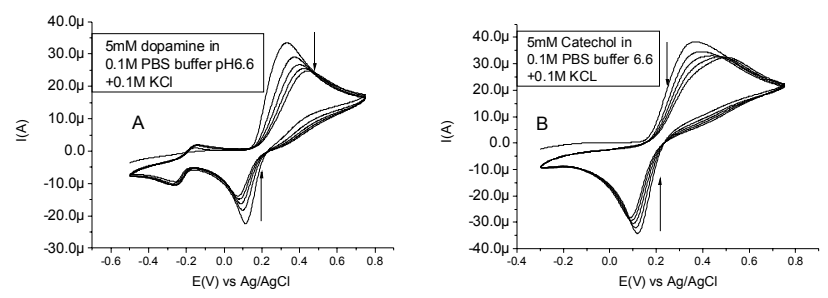

Figure 4: $\mathrm{CV}$ recorded in $5 \mathrm{mM}$ Dopamine (A) and catechol (B) in PBS Buffer pH6.6 (Au macro-electrode)
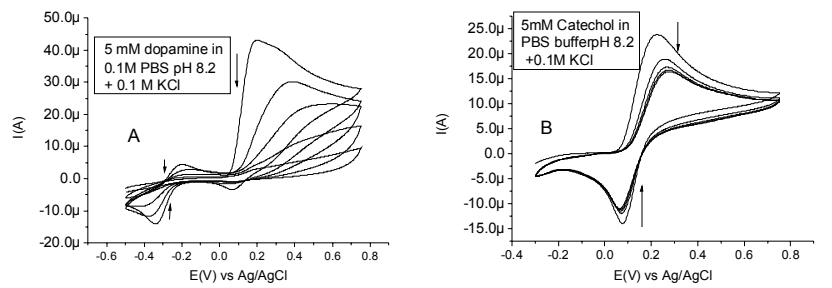

Figure 5: CV recorded in $5 \mathrm{mM}$ dopamine (A) and catechol (B) in PBS Buffer pH8.2 (Au macro-electrode)

The electrochemical oxidation of dopamine $(\mathrm{pKa}=8.92)$ has been suggested to be an ECC process. After electrochemical oxidation, dopamine loses two protons and two electrons and produces dopamine ortho-quinone. This intermediate undergoes intramolecular cyclization that latter polymerizes on the electrode surface. This reaction covers the surface with a melanin-like product and inhibits the electron transfer reaction.

In our experiments, we have used several buffers at different pHs $(3.9 ; 6.6 ; 8.2)$, in order to see the influence of solution acidity on the polymerization reaction. Successive cyclic voltammograms were recorded in $0.1 \mathrm{M}$ acetate buffer $(\mathrm{pH}$ 3.9) and $5 \mathrm{mM}$ dopamine and catechol (Figure 3). Two main peaks appear at about $+0.6 \mathrm{~V}$ and $+0.3 \mathrm{~V}$ and are due to the redox process for both dopamine and catechol. A slight decrease in the intensity of these peaks can be observed over 5 cycles. Another shoulder is evidenced at $-0.3 \mathrm{~V}$ for the dopamine and this is attributed to the polymer formed on the electrode surface.

Successive cyclic voltammograms recorded in PBS buffers $\mathrm{pH} 6.6$ and 8.2, showed a strong decrease of the anodic and cathodic peaks. Although dopamine is still protonated in 
these solutions, the polymerization process covers the electrode surface and hinders the electron transfer reaction (Figure 4 and Figure 5).

The pKa value for the catechol is 7 , so in the basic buffer solutions, a sharp peak of oxidation and reduction was observed. Although, a gradual decrease of the peaks were observed due to the deposition of polymer on the metal electrode $(\mathrm{Au})$.

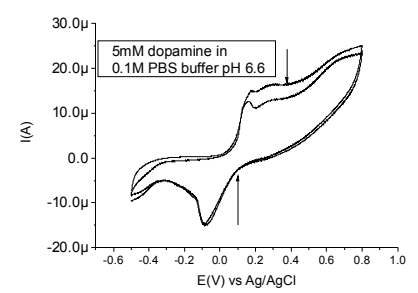

Figure 6: CV recorded using a thermally deposited Au micro-electrode- Reference electrode Ag/Ag/CI - Counter Electrode External Pt wire- $5 \mathrm{mM}$ dopamine in PBS buffer pH 6.6

The electrochemical detection of the CE-ECD has been tested with the actual gold micro electrode as a working electrode. It appears that the integrated $\mathrm{Ag} / \mathrm{AgCl}$ reference electrode allow to detect the two-electrons transfer during the oxidation of the dopamine (Figure 6). This first result opens wide range of molecular targets where complex samples could be processed and detected easily in a compact and disposable microchip.

\section{CONCLUSIONS}

A polymeric microchip capillary electrophoresis system with end-channel electrochemical detection using $\mathrm{Au}$ micro electrodes is reported. This work will lead to low cost and robust Lab-on-a-chip systems that have high sensitivity and selectivity for a wide range of applications. These first results show that the method appears to have good sensitivity in the same region of $\mathrm{pH}$. The limit of detection (LOD) and lower limit of quantification (LLOQ) of this method need to be assessed for both components and other molecules of interest. Optimization of the design and practical applications is in ongoing in our laboratory..

\section{ACKNOWLEDGMENT}

M. Rashid thanks the Centre for Nano \& Microsystems and Centre of Instrumentation in University of Teesside.

\section{REFERENCES}

[1] A. Manz, N.Graber, H.M. Widmer, Sens. Actuators B 1990, 1, $244-$ 248.

[2] A. Manz, D.J. Harrison, E. Verpoorte, et al., J. Chromatogr. 1992, 593, 253-258.

[3] K. Huikko, R. Kostiainen, T. Kotiaho, European J. Pharma. Science, 2003,20,149-171

[4] T. Vilkner, D. Janasek, A. Manz, Anal.Chem. 2004, 76, 3373-3386

[5] D.R. Reyes, D. Iossifidis, P.-A. Auroux, A. Manz, Anal. Chem. 2002, 74, 2623-2636.

[6] P.-A. Auroux, D. Iossifidis, D.R. Reyes, A. Manz, Anal.Chem. 2002, $74,2637-2652$.
[7] A.T.Woolley, K. Lao, A.N. Glazer, R.A. Mathies, Anal. Chem. 1998, 70, 684-688.

[8] D.J.Harrison, A. Manz, Z. Fan, et al., Anal. Chem. 1992, 64, 1926 1932.

[9] D.J. Harrison, K. Fluri, et al., Science. 1993, 261, 895-897.

[10]V. DolnÍk, S. Liu, S. Jovanovich, Electrophoresis. 2000, 21, 41-54

[11]H. Becker, C. Gartner, Electrophoresis. 2000, 21, 12-26.

[12] J. Rossier, F. Reymond, P.E. Michel, Electrophoresis. 2002, 23, 858867.

[13]H. Becker, L.E. Locascio, Talanta. 2002, 56, 267-287.

[14]D. Belder, M. Ludwig., Electrophoresis. 2003, 24, 3595-3606.

[15]H. Makamba, J.H. Kim, et al., Electrophoresis. 2003, 24, 3607-3619.

[16]C.D. Garcia, G. Engling, et al., Environ. Sci. Technol. 2005, 39, 618.

[17]W.R. Vandaveer, S.A. Pasas-Farmer, et al., Electrophoresis 2004, 25, 3528-3549.

[18] M. Li, Y.C. Lin, et al., Sens. Actuators B 2006, 117, 451-456

[19] Z. Zou, J. Kai, et al., Sens. Actuators A 2007, (Article is in press)

[20]J.C. Roulet, R.Volkel, et al., Anal. Chem. 2002, 74, 3400-3407.

[21]J.H. Qin, Y.S. Fung, et al., J. Chromatogr. A 2004, 1027, 223-229.

[22] W.R. Vandaveer, S.A. Pasas-Farmer et al., Electrophoresis 2002, 23, 3667-3677.

[23]R.S. Martin, K.L. Ratzlaff, B.H. Huynh, S.M. Lunte, Anal. Chem. 2002, 74, 1136-1143.

[24] J.Wang, M. Pumera, M.P, et al., Electrophoresis 2002, 23, 596-601.

[25] M.A.Schwarz, B. Galliker, et al., Analyst 2001, 126, 147-151.

[26]D. Voet, J.G. Voet, Biochemistry, John Wiley \& Sons, New York 1990, pp. 678-729.

[27]H. Becker, U. Heim, Sens. Actuators A 2001, 83, 249-135

[28]R.S. Martin, A.J. Gawron, S.M. Lunte, C.S. Henry, Anal. Chem. 2000, 72, 3196-3202

[29]Z.F. Chen, Y.H. Gao, J.M. Lin, R.G. Su, Y.J. Xie, Chromatogr. A 2004, 1038, 239.

[30] R.P. Baldwin, T.J. Roussel, et al., Anal. Chem. 2002, 74, 3690-3697.

[31]Z.H. Fan, D.J. Harrison, Anal. Chem. 1994, 66, 177-184

[32] P.B. Hietpas, K.M. Bullard, D.A. Gutman, A.G. Ewing, Anal. Chem. 1997, $69,2292-2298$.

[33] Y. Liu, J.C. Fanguy, J.M. Bledsoe, C.S. Henry, Anal. Chem. 2000, $72,5939-5944$. 